\title{
Imprinted tumor suppressor gene ARHI induces apoptosis correlated with changes in DNA methylation in pancreatic cancer cells
}

\author{
HONG YANG ${ }^{1}$, XINGQING LU $^{1}$, JIAMING QIAN ${ }^{1}$, FENGJI XU $^{2}$, YIQUN HU ${ }^{1}$, \\ YINHUA YU ${ }^{3}$, ROBERT C. BAST $\mathrm{Jr}^{4}$ and JINGNAN LI ${ }^{1}$
}

\begin{abstract}
${ }^{1}$ Department of Gastroenterology, Peking Union Medical College Hospital, Peking Union Medical College, Chinese Academy of Medical Sciences, Beijing 100730; ${ }^{2}$ Department of Urinary Surgery, Peking Hemujia Hospital, Beijing 100000; ${ }^{3}$ Department of Gynecology, Ob/Gyn Hospital of Fudan University, Shanghai 200011, P.R. China; ${ }^{4}$ Department of Experimental Therapeutics, University of Texas, M.D. Anderson Cancer Center, Houston 77030, TX, USA
\end{abstract}

Received March 4, 2010; Accepted May 28, 2010

DOI: 10.3892/mmr_00000301

\begin{abstract}
Aplesia Ras homologue member I (ARHI, DIRAS3) is a Ras-related imprinted growth inhibitory gene whose expression is down-regulated in the majority of breast and ovarian cancers. This study investigated the inhibitory function of ARHI in pancreatic cancer. Six pancreatic cancer cell lines, tumor xenografts in nude mice and 20 pancreatic cancer tissue sections were analyzed. ARHI is widely expressed in ductal and acinar cells of normal pancreatic tissue, but is down-regulated or lost in approximately $50 \%$ of pancreatic cancers. Aberrant methylation of the ARHI locus was found in five pancreatic cancer cell lines, which exhibited downregulation or loss of ARHI expression. Hypermethylation was detected in five cell lines $(5 / 5,100 \%)$ at $\mathrm{CpG}$ island $\mathrm{I}$, in two cell lines $(2 / 5,40 \%)$ at $\mathrm{CpG}$ island II and in four cell lines (4/5, $80 \%$ ) at $\mathrm{CpG}$ island III. Re-expression of ARHI significantly inhibited the growth of pancreatic cancer cells. This inhibition was associated with the induction of apoptosis. Treatment with the demethylating agent 5-aza-2'deoxycytidine (5-aza-dC) restored ARHI mRNA expression, inhibited cell growth and induced apoptosis in PANC-1 and P3 human pancreatic cancer cells in culture. In nu/nu mice, 5 -aza-dC also inhibited the growth of PANC-1 xenografts and induced apoptosis, as observed by TUNEL staining. These effects were associated
\end{abstract}

Correspondence to: Dr Jiaming Qian, Department of Gastroenterology, Peking Union Medical College Hospital, Peking Union Medical College, Chinese Academy of Medical Sciences, Beijing 100730, P.R. China

E-mail: qjiaming57@gmail.com

Abbreviations: ARHI, Aplesia Ras homolog member I; FBS, fetal bovine serum; 5-aza-dC, 5-aza-2'deoxycytidine; LOH, loss of heterozygosity; COBRA, combined bisulfite restriction analysis; RT-PCR, reverse transcription-PCR

Key words: ras homolog member I, pancreatic cancer, apoptosis, DNA methylation with the re-expression of ARHI protein. Therefore, ARHI may serve as a growth inhibitory gene in a significant fraction of pancreatic cancers. Re-expression of ARHI significantly induced the apoptosis of pancreatic cancer cells. A demethylation agent reduced human pancreatic cancer cell line growth in conjunction with ARHI re-expression.

\section{Introduction}

The ras oncogene serves as a molecular switch that alters signal transduction and promotes tumor cell proliferation, differentiation and invasion (1-3). It has been found that $72-90 \%$ of pancreatic cancers contain activating mutations in $K$-ras that are thought to play pivotal roles in initiating pancreatic ductal carcinogenesis (4-6). More than 100 GTP-binding proteins have been identified as members of the Ras superfamily $(7,8)$. The majority of Ras superfamily members are proto-oncogenes that promote the development of human cancer. However, Aplesia Ras homolog member I (ARHI, DIRAS3) is a small $26-\mathrm{kDa}$ GTPase that inhibits anchorage-dependent and -independent growth, motility, invasion and angiogenesis, despite sharing 54-62\% amino acid homology with Ras and Rap (9). Artificially induced expression of ARHI in mice causes small size, infertility and decreased lactation (10). Overexpression of ARHI in cancer cells that express low levels of ARHI triggers apoptosis through a caspase-independent, calpain-dependent mechanism $(12,13)$. Recent studies suggest that the re-expression of ARHI at physiologic levels also induces G2/M cell cycle arrest, autophagy and tumor dormancy in ovarian cancer (14).

ARHI is a maternally imprinted gene encoded by the second of two exons. It is expressed in several normal tissues, including tissues of the breast, ovary and pancreas. Downregulation of ARHI has been observed in $>60 \%$ of breast and ovarian cancers $(15,16)$. Decreased ARHI expression from the paternal allele occurs by several different mechanisms, including loss of heterozygosity, transcriptional regulation by E2F1 and E2F4, shortened mRNA half life and DNA methylation of multiple $\mathrm{CpG}$ islands with silencing of both alleles. 
Methylation occurs on two CpG islands (I and II) located in the promoter region and a third $\mathrm{CpG}$ island (CPG III) in the coding region of exon 2. In normal breast and ovary tissue, only the imprinted maternal allele is methylated, resulting in partial methylation for each of the three $\mathrm{CpG}$ islands. In $10 \%$ of breast and ovarian cancers, hypermethylation of both alleles is associated with loss of ARHI expression $(17,18)$.

The expression and function of ARHI in pancreatic cancer has received relatively little attention. As ARHI appears to oppose Ras function and K-ras is frequently activated in pancreatic cancers, it is possible that the loss of ARHI contributes to pancreatic carcinogenesis. In the present study, we measured the expression of ARHI in normal pancreas and pancreatic cancer tissue. The methylation status of ARHI was examined in pancreatic cancer cell lines with low ARHI expression. Pancreatic cancer cells with low ARHI expression were transfected with the pIRES2-EGFP-ARHI plasmid to examine the functional impact of ARHI re-expression on cancer cell growth in culture. A demethylating agent 5-aza-2'deoxycytidine (5-aza-dC), was tested for its ability to up-regulate ARHI mRNA expression in pancreatic cancer cells and to restore ARHI protein expression in human pancreatic cancer xenografts. The effect of ARHI expression on pancreatic xenograft growth was also measured.

\section{Materials and methods}

Cell lines. Five pancreatic cancer cell lines (PANC-1, PuPan-1, AsPC-1, SW1990 and MIA PaCa-2) were obtained from ATCC. The P3 pancreatic cancer cell line was established in the Pathology Department of Peking Union Medical College Hospital. All cells were grown in medium containing RPMI1640 supplemented with $10 \%$ fetal bovine serum (FBS), $100 \mathrm{mM}$ L-glutamine, $100 \mu \mathrm{g} / \mathrm{ml}$ streptomycin and $100 \mathrm{U} / \mathrm{ml}$ penicillin. Cells were grown to near confluence at $37^{\circ} \mathrm{C}$ in an atmosphere of $95 \%$ humidified air and $5 \% \mathrm{CO}_{2}$.

Patients and tissues. The study comprised 20 patients with primary pancreatic cancer that underwent initial surgery at PUMC hospital and Zhejiang Medical College Hospital between 1990 and 2001. Histopathologic diagnosis was based on the WHO criteria. The mean age of the 20 cases was 63 years (range 38-73). For the comparison of ARHI expression, 14 normal pancreas tissues were selected.

Immunohistochemical analysis. After initial deparaffinization of $4-\mu \mathrm{m}$ tissue sections, endogenous peroxidase activity was blocked using $0.3 \%$ hydrogen peroxide. Antigen retrieval was performed by heating the sections for $10 \mathrm{~min}$ in a microwave with $10 \mathrm{mM}$ sodium citrate ( $\mathrm{pH}$ 6.0). The slides were then incubated with monoclonal antibodies against ARHI. Slides were washed and incubated with streptavidin peroxidase-labeled secondary antibody for $1.5 \mathrm{~h}$ and then washed with phosphate buffered saline (PBS). Normal breast tissue was used as a positive control. PBS or the isotype matched (IgG1) anti-leukocyte common antigen monoclonal antibody was used in place of the primary anti-ARHI antibody as a negative control.

Transfection and measurement of cell apoptosis by flow cytometric analysis using propidium iodide staining. PANC-1 and MIA PaCa-2 cell lines were transfected with the pIRES2EGFP-ARHI constructs or with an empty pIRES2-EGFP vector as a control using Lipofectamine 2000 (Invitrogen)(19). The cells were placed in selection medium containing $900 \mu \mathrm{g} /$ ml G418 (Gibco BRL) for PANC-1 cells or $800 \mu \mathrm{g} / \mathrm{ml} \mathrm{G} 418$ for MIA PaCa-2 cells. Stable G418-resistant cell colonies were analyzed to detect ARHI protein expression by Western blotting (19).

Pancreatic cancer cells and their stable transfectants (pIRES2-EGFP-ARHI and pIRES2-EGFP) (1x10 $)$ were seeded in 6-well plates. To analyze apoptosis, transfected cells were collected and a single cell suspension was prepared in PBS. Cells were washed twice with cold PBS, fixed with $70 \%$ alcohol at $4^{\circ} \mathrm{C}$ for $24 \mathrm{~h}$, and stained with propidium iodide (PI; Sigma). Apoptosis was detected using flow cytometric analysis (Coulter Epics XL).

DNA/RNA extraction. Genomic DNA was extracted from the cell lines using the QIAamp DNA Mini kit (Qiagen). Total cellular RNA was extracted using TRIzol as described in the manufacturer's protocol (Gibco BRL).

Combined bisulfite restriction analysis (COBRA). Bisulfite treatment of DNA and COBRA was performed as described by Yuan et al (18). DNA was then precipitated and electrophoresed in $8 \%$ polyacrylamide gels. The gels were stained with ethidium bromide and the intensity of methylated alleles was calculated by densitometry using Image software (fluorchem 8800). A methylation density cutoff point of $15 \%$ was considered significant.

Treatment of cells with 5-aza-dC. Pancreatic cancer cell lines were suspended in RPMI-1640 with $10 \%$ FBS and allowed to attach over a 24-h period. Cells were treated with a final concentration of 0.4-1 $\mu \mathrm{M}$ 5-aza-dC (Sigma) and grown for up to 5 days. The medium, with or without 5 -aza-dC, was changed daily. At different times (24, 48, 72, 96 and 120 h), the medium was removed and MTT $(5 \mathrm{mg} / \mathrm{ml})$ was added to the wells. After $4 \mathrm{~h}$ at $37^{\circ} \mathrm{C}$, the media was removed and replaced with DMSO (150 $\mu \mathrm{l} / \mathrm{well})$. The plates were agitated at room temperature for $10 \mathrm{~min}$ and absorbance was read on an enzyme-linked immunosorbent assay reader at $490 \mathrm{~nm}$. Apoptosis was detected using PI staining and flow cytometry as described above. At the end of $120 \mathrm{~h}$, RNA and DNA were extracted for reverse transcription-PCR (RT-PCR) and bisulfite analysis.

Reverse transcription-PCR. Total cellular RNA was extracted using TRIzol as described in the manufacturer's protocol (Gibco). cDNA was synthesized using reverse transcriptase (Promega), $1 \mu \mathrm{g}$ total RNA, oligo(dT) and random primers (Promega). The PCR conditions and PCR primer sequences used were described by Yuan et al (18). The predicted PCR product size was $\sim 250 \mathrm{bp}$.

Tumor xenografts in nude mice. Five-week-old male athymic nude mice (Balb/c, nu/nu) were obtained from the Chinese Academy of Medical Sciences (Beijing, P.R. China). All mice had free access to sterilized food and autoclaved water. A suspension of PANC-1 cells $\left(1 \times 10^{6}\right.$ cells in $\left.0.1 \mathrm{ml} \mathrm{PBS}\right)$ 
A

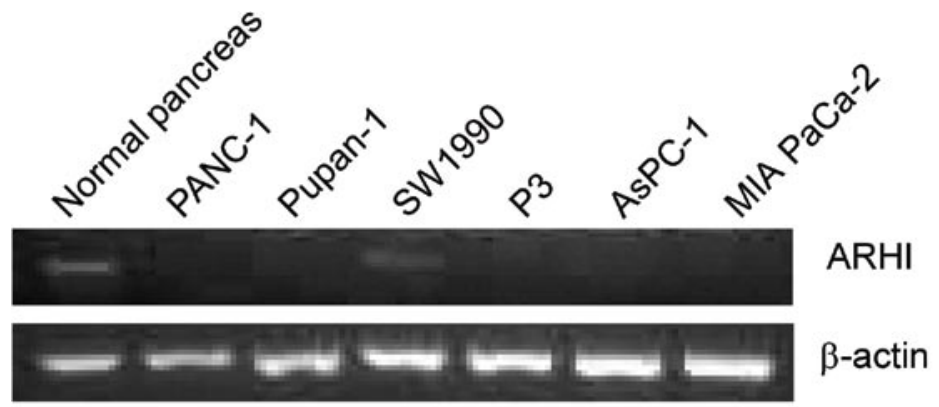

B

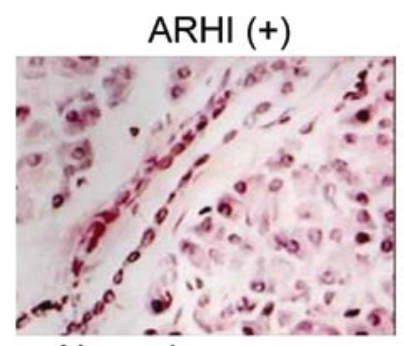

Normal pancreas
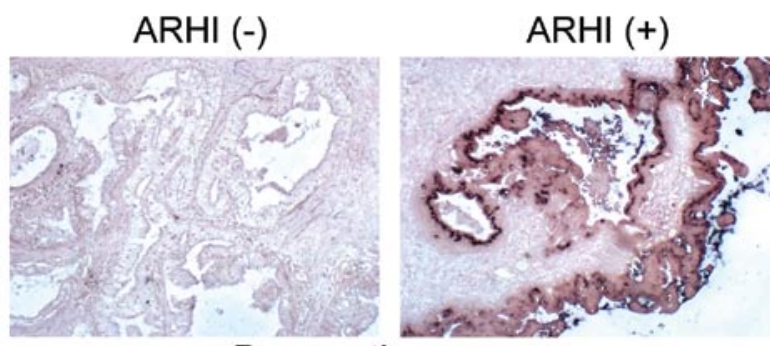

Pancreatic cancer

Figure 1. RT-PCR analysis of ARHI mRNA expression in pancreatic cancer cell lines and ARHI protein expression in normal pancreas and pancreatic cancer tissue by immunohistochemical staining. (A) ARHI mRNA expression was not detected in five of the six pancreatic cancer cell lines (PANC-1, P3, PuPan-1, AsPC-1 and MIA PaCa-2) by RT-PCR (above). Weak ARHI mRNA expression was observed in SW1990 cells. Normal pancreas was used as the positive control. $\beta$-actin was included as the loading control. (B) Immunohistochemical staining showed ARHI protein expression in normal pancreas and pancreatic cancer tissue (x15). The staining occurred primarily in the cytoplasm of normal cells. By contrast, ARHI protein expression was decreased or absent in 10 of $20(50 \%)$ pancreatic cancer tissue specimens.

was injected subcutaneously into the dorsal flank of each nude mouse. The animals were randomly divided into two groups when tumor size reached $4 \mathrm{~mm}$. One group of mice received subcutaneous injections of 5-aza-dC $(1 \mu \mathrm{g} / \mathrm{g})$ around the tumor and the second group of mice was injected with diluent-treated solution as controls. Tumor diameter was measured with a caliper ruler each day and tumor volume $\left(\mathrm{mm}^{3}\right)$ was estimated. On Days 3, 5, 7 and 9 after therapy, the mice were sacrificed and tumor tissue was fixed in $10 \%$ formalin for immunohistochemical examination using an anti-ARHI antibody as described below. Tumor cell apoptosis was judged by the TUNEL assay and performed as described in the manufacturer's protocol (Roche).

Statistical analysis. Data were expressed as the mean \pm SD. The Student's t-test and ANOVA were calculated using statistics software (SPSS 12.0) to determine the difference between the groups. A P-value of $<0.05$ was considered to be statistically significant.

\section{Results}

ARHI mRNA was down-regulated in the majority of pancreatic cancer cell lines and ARHI protein was found in normal pancreas tissue, but was decreased in $50 \%$ of pancreatic cancers. ARHI mRNA expression was not detected in five of the six pancreatic cancer cell lines (PANC-1, P3, PuPan-1, AsPC-1 and MIA PaCa-2) by RT-PCR. Weak ARHI mRNA expression was observed in SW1990 cells (Fig. 1). Immunohistochemical staining showed that ARHI protein was expressed in ductal epithelial, acinar and islet cells in 14 normal pancreas tissue specimens (Fig. 1). ARHI protein expression was primarily detected in the cytoplasm of normal cells, but was decreased or absent in 10 of $20(50 \%)$ pancreatic cancer tissue specimens (Fig. 1). There was no correlation between ARHI expression and cancer differentiation or clinical stage (data not shown).

Transfection of ARHI induced ARHI re-expression, which in turn induced apoptosis, but did not alter cell morphology. Our previous studies showed that the transfection of ARHI induced ARHI re-expression and inhibited the growth of pancreatic cancer cells (19). Inhibition of tumor cell growth by ARHI re-expression may result from cell cycle arrest, apoptosis, or a combination of both mechanisms. To evaluate the possible role of cell cycle arrest and apoptosis in ARHI-induced growth inhibition, PANC-1 and MIA PaCa-2 cells were transfected with pIRES2-EGFP-ARHI or pIRES2-EGFP. On Day 5, apoptosis was evaluated by flow cytometric analysis after staining of cellular DNA with PI. Increasing numbers of apoptotic PANC-1 cells (Fig. 2A; 32.94 \pm 15.06 vs. $13.78 \pm 6.36$ and $6.5 \pm 3.832 \%, \mathrm{P}<0.05$ ) and MIA PaCa-2 (Fig. 2B; 45.76 \pm 21.70 vs. $15.18 \pm 4.09$ and $8.08 \pm 1.97 \%, \mathrm{P}<0.05)$ were observed after transfection with pIRES2-EGFP-ARHI as compared to vector controls and parental cells. Transfection of both cell lines with pIRES2-EGFP-ARHI slightly increased the fraction of cells in the G0-G1 phase and decreased the fraction of cells in the $S$ phase (19). In addition, no significant change in cell morphology was observed after transfection with pIRES2EGFP-ARHI, as determined by fluorescence microscopy.

Hypermethylation of ARHI CpG islands was observed in five pancreatic cancer cell lines. Most early gene studies have suggested that only the methylation of $\mathrm{CpG}$ islands in 
A

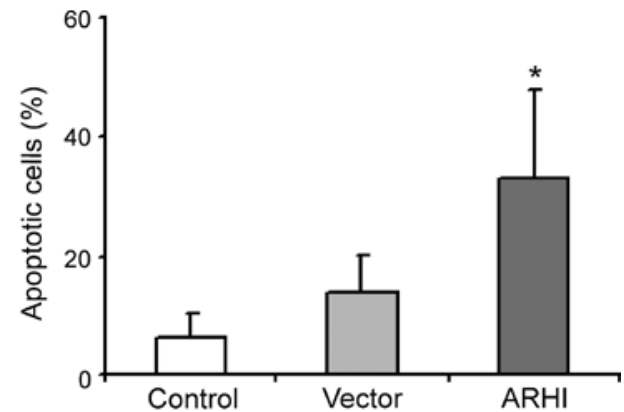

B

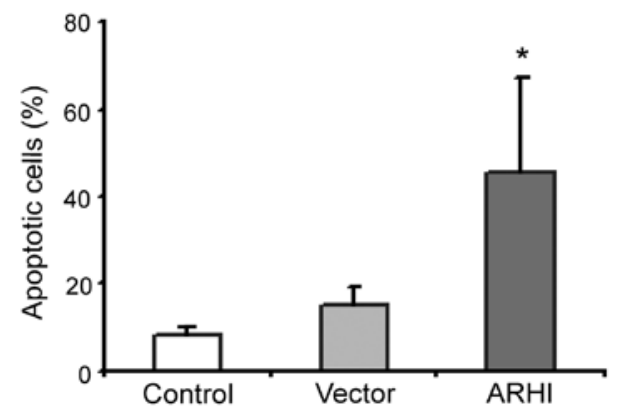

Figure 2. Effects of ARHI re-expression on apoptosis. PANC-1 (A), MIA $\mathrm{PaCa}-2$ (B) and their stable transfectants were examined for apoptosis. The apoptotic index of PANC-1 cells in the pIRES2-EGFP-ARHI group (ARHI) was significantly higher than pIRES2-EGFP (Vector) and the parental cell group (control) as determined by the Student's t-test $(32.94 \pm 15.06$ vs. $13.78 \pm 6.36$ and $6.5 \pm 3.832 \%)\left({ }^{*} \mathrm{P}<0.05\right)$. Similar results were observed in MIA PaCa-2 cells $(45.76 \pm 21.70$ vs. $15.18 \pm 4.09$ and $8.08 \pm 1.97 \%)\left({ }^{*} \mathrm{P}<0.05\right)$. The data shown are representative of at least five independent experiments.

the promoter region plays a role in repressing gene expression. Two potential CpG islands of $\sim 300$ bp each were found within the promoter of ARHI. In contrast to the consistent partial methylation (one allele) of ARHI in normal pancreatic tissue (Fig. 3A), hypermethylation (both alleles) was found at CpG island I in five pancreatic cancer cell lines $(100 \%)$. In two (40\%) of the cell lines (P3 and PANC-1), hypermethylation was observed at $\mathrm{CpG}$ island II (Fig. 3B). A methylation density cutoff point of $15 \%$ was considered significant. Values of $<15 \%$ were considered hypomethylated, $15-85 \%$ partially methylated and 85-100\% hypermethylated.

Treatment with a demethylating agent $(5-a z a-d C)$ reactivated $A R H I$ and reversed the methylation of ARHI CpG islands in pancreatic cancer cells. Two potential $\mathrm{CpG}$ islands of $\sim 300$ bp each were found within the promoter of ARHI. ARHI expression was lost and ARHI CpG islands I and II were hypermethylated in the PANC-1 and $\mathrm{P} 3$ pancreatic cancer cell lines. Treatment with 5-aza-dC partially restored the decrease in the methylation of the the CpG islands (I and II) (Fig. 4A) and restored ARHI expression in both lines (Fig. 4B).

Treatment with 5-aza-dC inhibited cell proliferation and induced apoptosis in PANC-1 and P3 pancreatic cancer cells. To determine the effect of DNA demethylation on pancreatic cancer cells, we measured cell growth and apoptosis in PANC-1 and P3 cells after treatment with 5-aza-dC. PANC-1 (Fig. 5A) and $\mathrm{P} 3$ cell growth (Fig. 5B) was significantly inhibited over time following treatment with 5 -aza-dC as compared to diluent treated controls. After 96 and 120 h, P3 growth was significantly

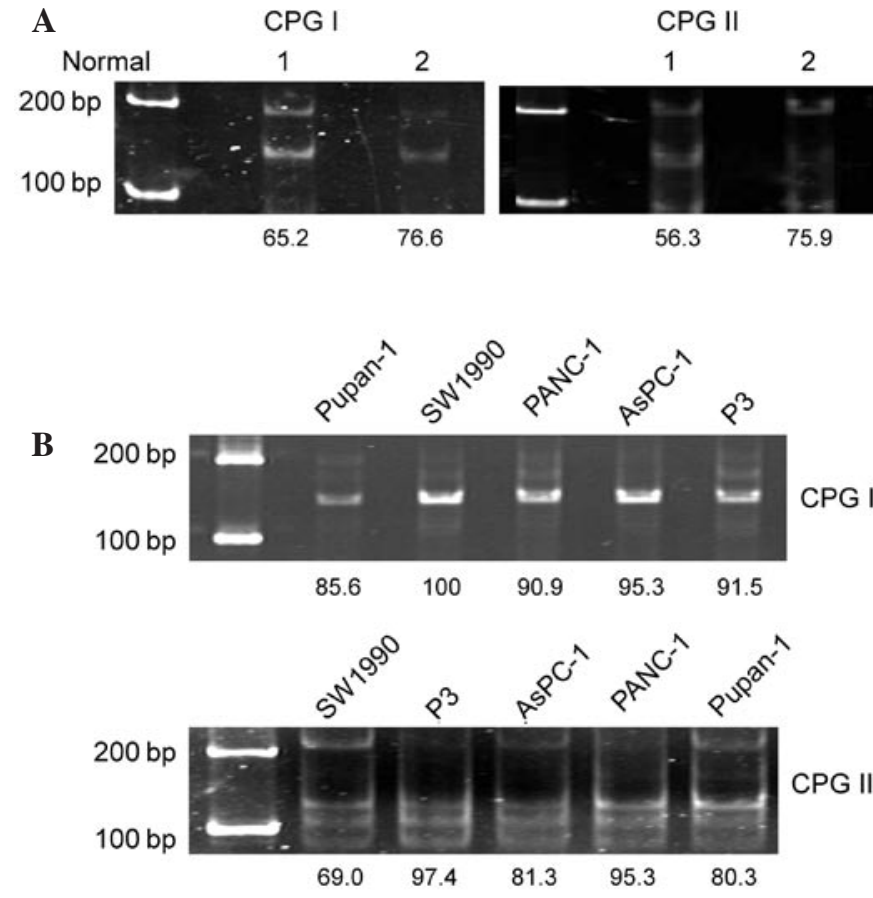

Figure 3. Methylation status of normal pancreas tissue and aberrant methylation of ARHI CpG islands in pancreatic cancer cell lines. Two normal pancreases showed partial methylation of ARHI (CpG I and II) (A). However, hypermethylation (both alleles) was found at $\mathrm{CpG}$ island I in five pancreatic cancer cell lines $(100 \%)$ and in two cell lines (40\%) at CpG island II (B). Methylation density (percentage of restricted vs. unrestricted fragment) was quantitated by densitometry and is shown below each lane.

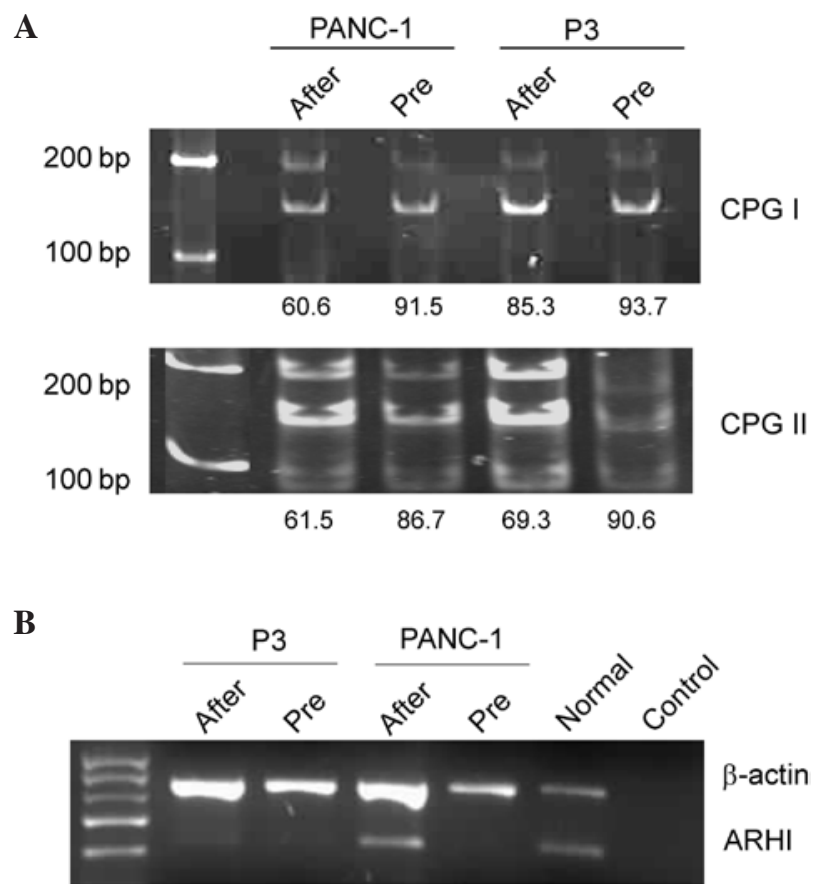

Figure 4. Effect of 5-aza-dC on DNA methylation of ARHI and ARHI gene mRNA expression in PANC-1 and P3 cells. Treatment with 5-aza-dC decreased hypermethylation to partial methylation in P3 and PANC-1 cells ( $\mathrm{CpG}$ islands I and II) (A). In addition, ARHI expression was restored in treated cells as compared to pre-treated cells (B).

reduced $(1.542 \pm 0.208$ and $1.734 \pm 0.222)$ following treatment with $1.0 \mu \mathrm{M} 5$-aza-dC as compared to the diluent-treated control 

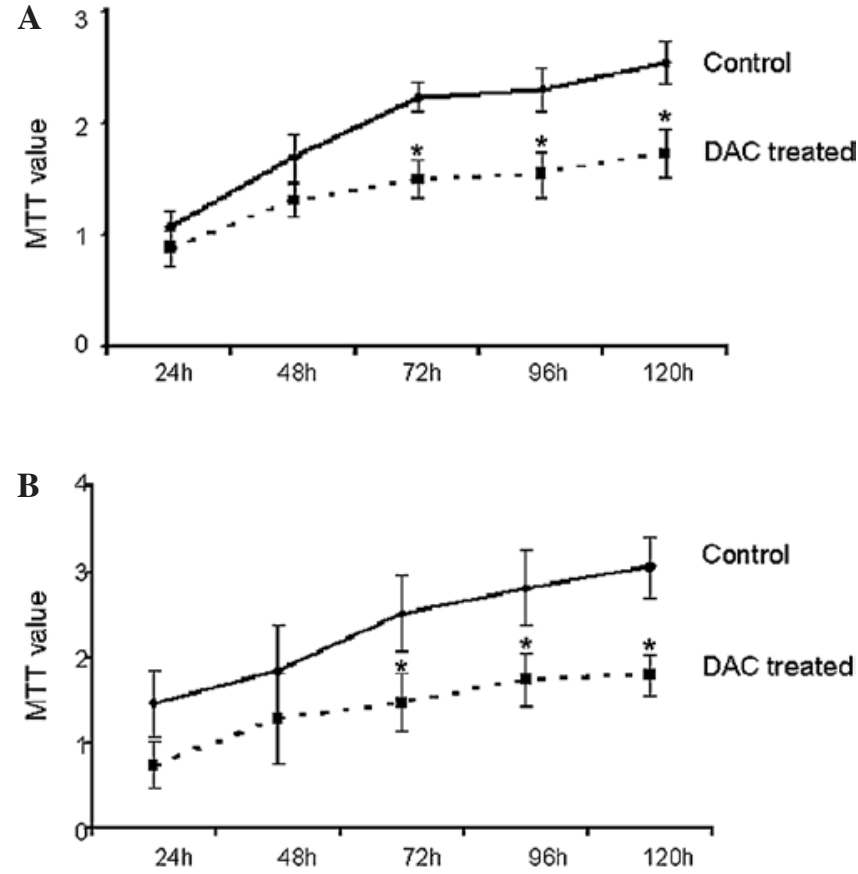

Figure 5. Effect of demethylation with 5-aza-dC on PANC-1 and P3 cell growth. PANC-1 (A) and P3 cells (B) were treated with 5-aza-dC or with diluent control. The average OD value at different intervals was measured. OD values reflect the mean number of three replicate wells at different intervals from three experiments (mean $\pm \mathrm{SD}, \mathrm{n}=3$ ). At $120 \mathrm{~h}$, P3 and PANC-1 cell growth was significantly reduced $(1.734 \pm 0.222$ and $1.732 \pm 0.311)$ following treatment with $1.0 \mu \mathrm{M} 5$-aza-dC as compared to the diluent-treated control $(2.541 \pm 0.196$ and $3.051 \pm 0.346)(\mathrm{P}<0.05)$.

Table I. Apoptotic index in PANC-1 and P3 cells with $1.0 \mu \mathrm{M}$ decitabine treatment.

\begin{tabular}{lccc}
\hline Cells & $\begin{array}{c}\text { Control } \\
(\text { mean } \pm \text { SD } \%)\end{array}$ & $\begin{array}{c}\text { Treatment group } \\
(\text { mean } \pm \text { SD } \%)\end{array}$ & P-value \\
\hline P3 & $1.85 \pm 0.12$ & $20.56 \pm 8.29^{\mathrm{a}}$ & 0.003 \\
PANC-1 & $2.43 \pm 1.00$ & $32.77 \pm 10.38^{\mathrm{a}}$ & 0.001 \\
\hline
\end{tabular}

Induction of apoptosis with 5-aza-dC treatment in P3 and PANC-1 cells on Day 5. Apoptosis was measured by PI staining using FACS. The apoptosis index was measured three times. The apoptosis index of P3 and PANC-1 cells in the treatment group was significantly higher than in the diluent-treated control $\left({ }^{a} \mathrm{P}<0.05\right)$, expressed as the mean from three experiments (mean $\pm \mathrm{SD}, \mathrm{n}=3$ ).

(2.297 \pm 0.193 and $2.541 \pm 0.196)(\mathrm{P}<0.05)$. Similar differences were noted in PANC-1 cells treated with $1.0 \mu \mathrm{M} 5$-aza-dC at $96 \mathrm{~h}(1.732 \pm 0.311$ vs. $2.813 \pm 0.4343)$ and $120 \mathrm{~h}(1.798 \pm 0.242$ and 3.051 \pm 0.346$)(\mathrm{P}<0.05)$. To evaluate the role of apoptosis in 5-aza-dC-induced growth inhibition, apoptosis was evaluated by flow cytometric analysis after staining of cellular DNA with PI. An increased number of apoptotic PANC-1 and P3 cells was observed on Day 5 after 5-aza-dC treatment as compared to the diluent-treated control $(\mathrm{P}<0.01)$ (Table I).

5-aza-dC treatment increases ARHI expression and induces apoptosis in human pancreatic cancer xenografts. We
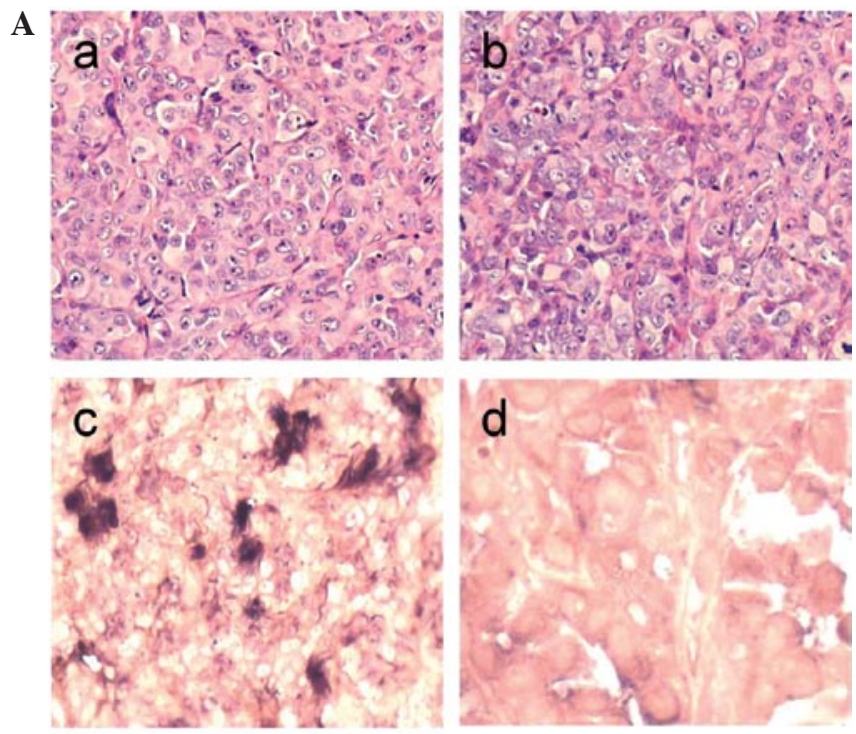

B
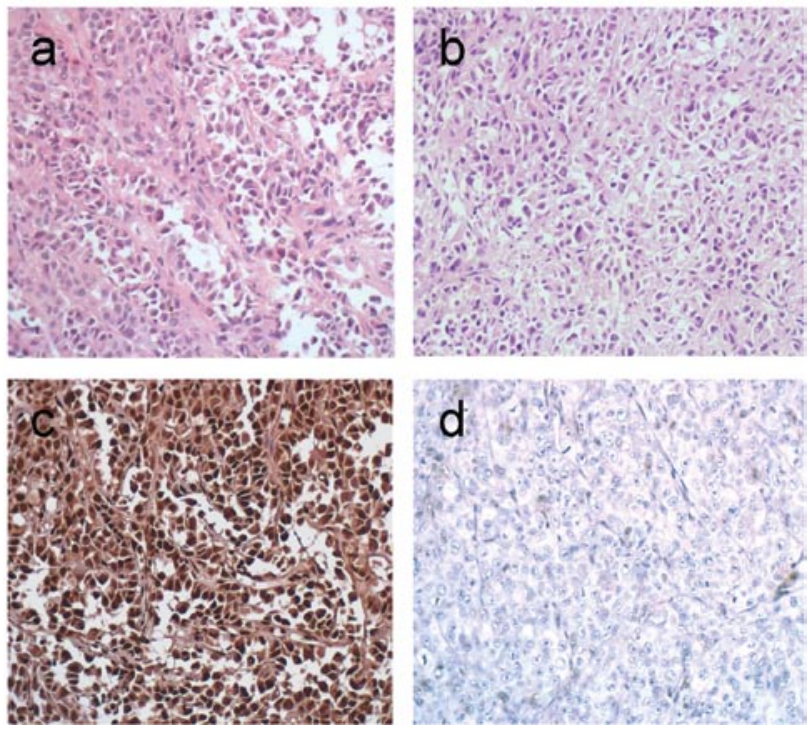

Figure 6. Effect of 5-aza-dC on ARHI protein expression and cell apoptosis in nude mice tumor xenografts on Day 3 following injection with 5-aza-dC. (A) ARHI protein was partly restored in the treatment group but not in the diluent-treated control group (x20). H\&E in tumor xenografts (a); positive ARHI protein expression in the same case as ' $a$ ' (c); H\&E in tumor xenografts (b); negative ARHI protein expression in the same case as 'b' (d). (B) Apoptotic nuclei appeared after 5-aza-dC treatment in PANC-1 xenografts as judged by TUNEL staining (x15). H\&E staining in treatment group (a); TUNEL staining in treatment group (c); H\&E staining in dilute control group (b); TUNEL staining in dilute control group (d).

examined the effect of the 5-aza-dC demethylating agent on ARHI expression in vivo. PANC-1 cells were injected in the right flanks of 5-week-old nude mice. The animals were randomly assigned to groups treated with 5 -aza-dC $(1 \mu \mathrm{g} / \mathrm{g})$ or diluent. The mice were sacrificed at 3,5,7 and 9 days following 5-aza-dC injection. Treatment with 5-aza-dC restored ARHI protein expression after 3 days as compared to the control (Fig. 6A). In addition, the diameter of the tumor was measured with a caliper ruler each day and the volume of the tumor $\left(\mathrm{mm}^{3}\right)$ was estimated. The tumor volume decreased significantly in the 5-aza-dC treatment group compared to the control group. The rates of tumor inhibition were 52.7, 78.9, 78.2 and $97.1 \%$, respectively, after treatment with 5 -aza-dC on different days (3, 
5, 7 and 9). 5-aza-dC increased the number of apoptotic cells in xenografts as compared to the controls (Fig. 6B).

\section{Discussion}

Pancreatic carcinogenesis is a multistep process during which oncogenes are activated and the function of tumor suppressor genes is lost (20-22). K-ras mutation, telomere shortening, p16 loss, p53 loss and smad4 loss are thought to contribute to pancreatic carcinogenesis, but $K$-ras mutation is the most prevalent mechanism associated with this cancer. This is the first report showing that ARHI expression is lost in approximately half of pancreatic cancers. Notably, ARHI is an imprinted tumor suppressor gene that has $60 \%$ homology to Ras and opposes Ras gene function. We showed that ARHI is widely expressed in the cytoplasm of normal pancreatic ducts and acinar cells, but is dramatically down-regulated in 50\% of pancreatic cancers. Consequently, the loss of ARHI function is likely to be one of the most prevalent events affecting tumor suppressor genes in pancreatic cancer. We did not find any correlation between ARHI expression and clinical parameters, but this lack is likely due to the limited number of cases studied.

Re-expression of ARHI through plasmid transfection inhibited pancreatic cancer cell proliferation and suppressed clonogenic growth. Re-expression of the ARHI gene triggered apoptosis in PANC-1 and MIA PaCa-2 cells. Bao et al observed cell cycle arrest and apoptosis in ovarian cancer cell lines after ARHI re-expression and found that it relates to caspase-independent and calpain-dependent apoptotic events (12). In pancreatic cancer cells, our data showed that cancer cell apoptosis was related to DAPK1-dependent events (unpublished data). Thus, the ARHI gene appears to have growth inhibitory activity in pancreatic, breast and ovarian cancers.

Previous studies by Yu et al showed strong ARHI mRNA expression in normal pancreas, weak ARHI expression in liver and no ARHI expression in colon. Our previous studies found no detectable ARHI expression in normal Ges-1 cells (unpublished data). Loss of ARHI expression is not a universal event in gastrointestinal cancer, but it may be important in pancreatic oncogenesis.

Multiple mechanisms may contribute to the down-regulation of ARHI expression, including LOH, DNA methylation and transcriptional regulation. ARHI is an imprinted gene and its maternal copy is silenced by methylation. ARHI is expressed only from the paternal allele in all normal cells. As suggested, the methylation of $\mathrm{CpG}$ islands in the promoter region of ARHI plays a role in repressing gene expression. In this study, we observed aberrant methylation of ARHI in the promoter region from five pancreatic cancer cell lines. All cell lines showed hypermethylation of $\mathrm{CpG}$ island I (100\%), and two of them showed hypermethylation at $\mathrm{CpG}$ island II (40\%). PANC-1 and P3 cells showed hypermethylation at CpG islands I and II. SW1990 cells showed hypermethylation at $\mathrm{CpG}$ island I and partial methylation at $\mathrm{CpG}$ island II, which contributes to weak ARHI mRNA expression. AsPC-1 and PuPan-1 cells showed partial methylation at $\mathrm{CpG}$ island II and hypermethylation at $\mathrm{CpG}$ island I. These data suggest that aberrant methylation frequently occurs in pancreatic cancer cell lines. Together with previous studies in ovarian and breast cancer $(9,17,18)$, our observations support the potential role of aberrant methylation in the silencing of ARHI in multiple malignancies.

The possible role of ARHI in the growth regulation of pancreatic cancer was supported by the reactivation of $A R H I$ mRNA expression when PANC-1 and P3 cells were exposed to the 5-aza-dC demethylation agent; this effect was associated with the partial DNA methyaltion of only one allele. ARHI protein expression was detected in xenografts after 5-aza-dC treatment, but was not detectable following treatment with the diluent-treated control. Re-expression of ARHI was associated with the inhibition of xenograft growth and the induction of apoptosis. Treatment with the demethylation agent inhibited human pancreatic cancer cell growth and was accompanied by ARHI re-expression. 5-aza-dC treatment causes DNA damage and up-regulates other silenced genes, suggesting that other mechanisms may be involved.

The growth regulatory activity of ARHI and its loss in half of pancreatic cancers suggest that the loss of ARHI could be an important event in the pathogenesis of pancreatic cancer. Since one allele is silenced in normal pancreatic cells, loss of ARHI expression may occur in a single genetic event. Additional studies are required to further elucidate the role of ARHI at this site.

\section{Acknowledgements}

This study was supported by the National Natural Science Foundation of China (30670963), the Beijing Natural Science Foundation (7072062) and the Doctoral Fund of the Ministry of Education of China (20060023016).

\section{References}

1. Smakman N, Borel Rinkes IH, Voest EE and Kranenburg O: Control of colorectal metastasis formation by K-Ras. Biochim Biophys Acta 1756: 103-114, 2005.

2. Kranenburg O: The KRAS oncogene: past, present, and future. Biochim Biophys Acta 1756: 81-82, 2005.

3. Schubbert S, Shannon K and Bollag G: Hyperactive Ras in developmental disorders and cancer. Nat Rev Cancer 7: 295-308, 2007.

4. Hingorani SR, Petricoin EF, Maitra A, et al: Preinvasive and invasive ductal pancreatic cancer and its early detection in the mouse. Cancer Cell 4: 437-450, 2003.

5. Aguirre AJ, Bardeesy N, Sinha M, Lopez L, Tuveson DA, Horner J, Redston MS and DePinho RA: Activated Kras and Ink4a/Arf deficiency cooperate to produce metastatic pancreatic ductal adenocarcinoma. Genes Dev 17: 3112-3126, 2003.

6. Deramaudt T and Rustgi AK: Mutant KRAS in the initiation of pancreatic cancer. Biochim Biophys Acta 1756: 97-101, 2005.

7. Bourne HR, Sanders DA and McCormick F: The GTPase superfamily: conserved structure and molecular mechanism. Nature 349: 117-127, 1991.

8. Bos JL: ras oncogenes in human cancer: a review. Cancer Res 49: 4682-4689, 1989.

9. Yu Y, Xu F, Peng H, Fang X, Zhao S, Li Y, Cuevas B, Kuo WL, Gray JW, Siciliano M, Mills GB and Bast RC Jr: NOEY2 (ARHI), an imprinted putative tumor suppressor gene in ovarian and breast carcinomas. Proc Natl Acad Sci USA 96: 214-219, 1999.

10. Xu F, Xia W, Luo RZ, Peng H, Zhao S, Dai J, Long Y, Zou L, Le W, Liu J, Parlow AF, Hung MC, Bast RC Jr and Yu Y: The human ARHI tumor suppressor gene inhibits lactation and growth in transgenic mice. Cancer Res 60: 4913-4920, 2000.

11. Shi Z, Zhou X, Xu L, Zhang T, Hou Y, Zhu W and Zhang T: NOEY2 gene mRNA expression in breast cancer tissue and its relation to clinicopathological parameters. Zhonghua Zhong Liu Za Zhi 24: 475-478, 2002. 
12. Bao JJ, Le XF, Wang RY, Yuan J, Wang L, Atkinson EN, LaPushin R, Andreeff M, Fang B, Yu Y and Bast RC Jr: Reexpression of the tumor suppressor gene ARHI induces apoptosis in ovarian and breast cancer cells through a caspaseindependent calpain-dependent pathway. Cancer Res 62: 7264-7272, 2002.

13. Lu Z, Luo RZ and Khare S: Inducible expression of tumor suppressor gene ARHI in ovarian cancer cells triggers G2M arrest and inhibits cancer cell growth in vitro and in vivo. Proc Am Assoc Cancer Res 46: 2895, 2005.

14. Lu Z, Luo RZ, Lu Y, Zhang X, Yu Q, Khare S, Kondo S, Kondo Y, Yu Y, Mills GB, Liao WS and Bast RC Jr: The tumor suppressor gene ARHI regulates autophagy and tumor dormancy in human ovarian cancer cells. J Clin Invest 118: 3917-3929, 2008.

15. Yu Y, Luo R, Lu Z, Feng W, Badgwell D, Issa JP, Rosen DG, Liu J and Bast RC Jr: Biochemistry and biology of ARHI (DIRAS3), an imprinted tumor suppressor gene whose expression is lost in ovarian and breast cancers. Methods Enzymol 407: 455-468, 2006

16. Luo RZ, Fang X, Marquez R, Liu SY, Mills GB, Liao WS, Yu Y and Bast RC Jr: ARHI is a Ras-related small G-protein with a novel N-terminal extension that inhibits growth of ovarian and breast cancers. Oncogene 22: 2897-2909, 2003.
17. Luo RZ, Peng H, Xu F, Bao J, Pang Y, Pershad R, Issa JP, Liao WS, Bast RC Jr and Yu Y: Genomic structure and promoter characterization of an imprinted tumor suppressor gene ARHI. Biochim Biophys Acta 1519: 216-222, 2001.

18. Yuan J, Luo RZ, Fujii S, Wang L, Hu W, Andreeff M, Pan Y, Kadota M, Oshimura M, Sahin AA, Issa JP, Bast RC Jr and $\mathrm{Yu}$ Y: Aberrant methylation and silencing of ARHI, an imprinted tumor suppressor gene in which the function is lost in breast cancers. Cancer Res 63: 4174-4180, 2003.

19. Lu X, Qian J, Yu Y, Yang H and Li J: Expression of the tumor suppressor ARHI inhibits the growth of pancreatic cancer cells by inducing G1 cell cycle arrest. Oncol Rep 22: 635-640, 2009.

20. Baylin SB and Ohm JE: Epigenetic gene silencing in cancer a mechanism for early oncogenic pathway addiction? Nat Rev Cancer 6: 107-116, 2006.

21. Sato N and Goggins M: The role of epigenetic alterations in pancreatic cancer. J Hepatobiliary Pancreat Surg 13: 286-295, 2006.

22. Ghaneh P, Costello E and Neoptolemos JP: Biology and management of pancreatic cancer. Gut 56: 1134-1152, 2007. 